\title{
Short communication: Pseudomonas azotoformans causes gray discoloration in HTST fluid milk
}

\author{
Rachel L. Evanowski, Samuel J. Reichler, David J. Kent, Nicole H. Martin, Kathryn J. Boor, \\ and Martin Wiedmann ${ }^{1}$ \\ Milk Quality Improvement Program, Department of Food Science, Cornell University, Ithaca, NY 14853
}

\begin{abstract}
Pseudomonas species are well recognized as dairy product spoilage organisms, particularly due to their ability to grow at refrigeration temperatures. Although Pseudomonas-related spoilage usually manifests itself in flavor, odor, and texture defects, which are typically due to production of bacterial enzymes, Pseudomonas is also reported to cause color defects. Because of consumer complaints, a commercial dairy company shipped 4 samples of high temperature, short time (HTST)-pasteurized milk with distinctly gray colors to our laboratory. Bacterial isolates from all 4 samples were identified as Pseudomonas azotoformans. All isolates shared the same partial $16 \mathrm{~S}$ rDNA sequence and showed black pigmentation on Dichloran Rose Bengal Chloramphenicol agar. Inoculation of one pigment-producing $P$. azotoformans isolate into HTST-pasteurized fluid milk led to development of gray milk after $14 \mathrm{~d}$ of storage at $6^{\circ} \mathrm{C}$, but only in containers that had half of the total volume filled with milk $(\sim 500 \mathrm{~mL}$ of milk in $\sim 1,000$-mL bottles). We conclusively demonstrate that Pseudomonas can cause a color defect in fluid milk that manifests in gray discoloration, adding to the palette of color defects known to be caused by Pseudomonas. This information is of considerable interest to the dairy industry, because dairy processors and others may not typically associate black or gray colors in fluid milk with the presence of microbial contaminants but rather with product tampering (e.g., addition of ink) or other inadvertent chemical contamination.
\end{abstract}

Key words: gray pigment, spoilage, Pseudomonas, milk

\section{Short Communication}

Gram-negative Pseudomonas spp. are commonly associated with post-pasteurization contamination of milk (Dogan and Boor, 2003; Stellato et al., 2017).

Received January 26, 2017.

Accepted June 4, 2017.

${ }^{1}$ Corresponding author: mw16@cornell.edu
Pseudomonas spp., which grow well at refrigeration temperatures, can cause dairy product spoilage through a variety of mechanisms (Martin et al., 2011; Stellato et al., 2017). For example, many Pseudomonas spp. produce proteases and lipases, which can create sensory defects in food products (Dogan and Boor, 2003). Dogan and Boor (2003) reported that among 338 Pseudomonas isolates collected from raw milk, pasteurized milk, and dairy processing plant environments, 51\% were protease positive, $47 \%$ were lecithinase positive, and $67 \%$ were lipase positive. Some Pseudomonas spp. also produce several different pigments (Palleroni, 1984), which can cause discoloration of dairy products and other foods (Cantoni et al., 2003). Of particular relevance to dairy products, Pseudomonas fluorescens biovar IV produces both a blue nondiffusible pigment as well as soluble pyoverdin (Palleroni, 1984). Contamination of a commercial fresh, low-acid cheese with $P$. fluorescens biovar IV generated alarming dark blue and fluorescent pigments on the surface of the cheese (Martin et al., 2011); this color defect precipitated at least one product recall. This represents a major business risk, particularly in the modern era of social media, because consumers easily notice color defects. Images of products with color defects can be shared widely and quickly, rapidly fueling consumer backlash against a product or company.

As part of the routine services offered by Cornell University's Milk Quality Improvement Program (MQIP), we received 4 samples (in $60-\mathrm{mL}$ dairy vials) of fluid milk following consumer complaints of a "gray color defect." Samples were from whole, 2\%, and 1\% HTSTprocessed milk, and the gray discoloration had been an ongoing problem for the plant for several weeks before they contacted the MQIP. Microbiological analyses revealed large numbers of black-pigmented colonies on Dichloran Rose-Bengal Chloramphenicol (DRBC) agar; bacterial numbers were not assessed due to the unknown temperature history of the samples upon receipt. The same milk samples plated on SPC agar yielded gray colonies that produced pigment that diffused into the agar. Based on these initial observations, we hypothesized that the predominant dark-pigmented colonies 
isolated from the original milk samples were responsible for the observed gray color defect in the fluid milk. One pigmented isolate from each milk sample was characterized by partial 16S rDNA sequencing using previously described methods (Huck et al., 2007); all 4 isolates showed 100\% identity over 669 nucleotides of partial $16 \mathrm{~S}$ rDNA sequence data, suggesting that these isolates were clonal. Analysis of full-length $16 \mathrm{~S}$ rDNA sequences for one isolate (FSL E2-0548), which was selected for further characterization, classified this isolate as Pseudomonas azotoformans. Among the six 16S rRNA operon sequences obtained from the FSL E2-0548 genome sequence, 3 showed a top match (99.6\% identity) with the 16S rRNA sequence of the Pseudomonas azotoformans type strain included in the Ribosomal Database Project (RDP) Release 11 (https://rdp.cme.msu.edu). The FSL E2-0548 16S rRNA operon sequences also clustered with the 16S rRNA sequence of the Pseudomonas azotoformans type strain on a maximum likelihood phylogeny constructed with $16 \mathrm{~S}$ rDNA type strain sequences obtained from RDP. Therefore, we conclude that the predominant contaminant of the original milk samples was $P$. azotoformans. All bacterial isolates are maintained as frozen stocks and are available for future studies through the Cornell University Food Safety Laboratory collection (http://www.foodmicrobetracker .com). Further characterization of these isolates with whole-genome sequencing will provide additional information, including confirmation that the same strain was isolated from all samples, as well as potential information on pathways responsible for the formation of the molecules responsible for the gray color.

We selected a single dark pigment-producing $P$. azotoformans isolate (FSL E2-0548) for inoculation studies into HTST-pasteurized fluid milk to determine if this strain could cause the gray discoloration defect that was initially reported in the commercial fluid milk samples. Briefly, isolate FSL E2-0548 was streaked onto brain heart infusion (BHI) agar from a frozen glycerol stock and incubated for $48 \mathrm{~h}$ at $32^{\circ} \mathrm{C}$. A single colony was suspended in $5 \mathrm{~mL}$ of sterile BHI broth, followed by incubation for $18 \mathrm{~h}$ at $32^{\circ} \mathrm{C}$. After stationary incubation, $1 \mathrm{~mL}$ of the $10^{8} \mathrm{cfu} / \mathrm{mL}$ BHI suspension was placed in a $1.5-\mathrm{mL}$ microcentrifuge tube and centrifuged at $21,000 \times g$ for $1 \mathrm{~min}$. The pellet was resuspended in $1 \mathrm{~mL}$ of sterile PBS. Serial dilutions of the bacterial suspension were prepared in PBS and used to inoculate containers of homogenized and pasteurized $2 \%$ fat milk (Cornell University Dairy, Ithaca, NY) to a final concentration of approximately $10 \mathrm{cfu} / \mathrm{mL}$. Treatments groups included (1) inoculated "full" quart-size containers with original milk volume, (2) inoculated "half-full" quart-size containers with approximately half of the milk volume removed (to create headspace to simulate consumer usage in the home), as well as corresponding "full" and "half-full" uninoculated controls (3 and 4); each treatment was performed in 2 biological replicates. Five containers of each of the above treatments were prepared, one for each day of testing. Our goals were to ensure similar headspace and fluid milk volume for each sampling day and to prevent inadvertent bacterial contamination during subsequent handling.

The milk samples were stored at $6^{\circ} \mathrm{C}$. One container from each of the 4 treatment groups was tested at 0,3 , 7, 10, and $14 \mathrm{~d}$ after inoculation. Before analysis, each sample was mixed by 25 inversions, according to Standard Methods for the Examination of Dairy Products procedures for preparing fluid milk samples for microbiological analyses (Laird et al., 2004). Samples were spiral plated in duplicate, on the test days stated above, onto $100-\mathrm{mm}$ SPC agar plates using the $50-\mu \mathrm{L}$ exponential setting (Autoplate 5000, Advanced Instruments Inc., Norwood, MA). The plates were incubated for 48 $\mathrm{h}$ at $32^{\circ} \mathrm{C}$, and colonies were enumerated on the Color Q-Count (model 530, Advanced Instruments Inc.). To monitor possible color changes in the milk, $\mathrm{L}^{*} \mathrm{a} * \mathrm{~b}^{*}$ values were measured on each testing day for each sample using the D65/10 setting on a colorimeter (HunterLab ColorQuest XE, Hunter Associates Laboratory Inc., Reston, VA), and Euclidean distance $d_{w}$ from white was calculated by $d_{w}=\sqrt{\left(\mathrm{L}^{*}-100\right)^{2}+\left(\mathrm{a}^{*}\right)^{2}+\left(\mathrm{b}^{*}\right)^{2}}$, where $\mathrm{L}^{*}$ represents lightness $\left(\mathrm{L}^{*}=0\right.$ is black; $\mathrm{L}^{*}=100$ is a diffuse white); $a^{*}$ measures the sample's position between red/magenta and green (negative values are green; positive values are magenta); $b^{*}$ measures the sample's position between yellow and blue (negative values are blue; positive values are yellow) (Yam and Papadakis, 2004). A linear model was fit to model $d_{w}$ by time, headspace, and inoculation, including all interactions, in R (R Core Team, 2016). The lsmeans package (Lenth, 2016) was used to compare the fitted $d_{w}$ on $\mathrm{d} 14$ at different values of headspace or inoculation. Plots were generated with the ggplot package (Wickham, 2009).

"Half-full" fluid milk samples inoculated with P. azotoformans (FSL E2-0548) developed a gray color after 14 $\mathrm{d}$ of incubation at $6^{\circ} \mathrm{C}$ (Figure 1), whereas none of the uninoculated samples, with or without extra headspace, showed visibly detectable color development. Statistical analyses showed that the color (i.e., $d_{w}$ ) in the half-full inoculated bottles was significantly less white than the half-full controls $(P<0.0001)$. Additionally, the color in the half-full inoculated samples was significantly less white compared with the corresponding full containers $(P<0.0001)$, suggesting that oxygen availability plays 
a role in color development. At d 14, the visually gray milk from the inoculated half-full containers had an average $L^{*}$ value of 88.8 compared with 94.0 for the corresponding uninoculated milk, indicating darker colors in the gray milk (Figure 2A). Furthermore, the visually gray milk showed an average a* value of -1.8 , whereas the corresponding uninoculated milk showed an average $\mathrm{a}^{*}$ value of -0.61 , indicating a color shift toward green for the inoculated milk. The $\mathrm{b}^{*}$ values averaged 1.7 for the gray milk (in half-full containers) compared with 7.7 in the corresponding controls; this shift toward negative values is associated with blue color. Interestingly, bacterial counts did not differ between fluid milk samples in half-full containers and the corresponding samples in full containers; in all inoculated samples, bacterial counts were $>10^{8} \mathrm{cfu} / \mathrm{mL}$ by d 10 (Figure $2 \mathrm{~B})$. These results suggest that the development of gray color only in the half-full containers is not due to higher bacterial numbers under these conditions. Rather, these results suggest differences in bacterial metabolism or pigment chemistry in the full and half-full containers, likely due to the relative amount of atmospheric oxygen present in a given container.

Pseudomonas spp. have been shown to produce many pigmented compounds, such as pyocyanin (blue) and pyoverdin (fluorescent green; Palleroni, 1984). Pyocyanin pigment production has been reported to be oxygen dependent, and stationary-phase Pseudomonas aeruginosa can reduce pyocyanin from the oxidized blue form to a colorless form in the absence of oxygen (Price-
Whelan et al., 2007). We believe that pyocyanin was the most likely cause of the gray color defect, because the color was only found in the half-full containers, which had more headspace and, therefore, more oxygen. We hypothesize that $P$. azotoformans reduced pyocyanin to its colorless form in the full containers (with less oxygen), consistent with what has been reported for $P$. aeruginosa. Partially filled containers of milk have been previously reported to exacerbate sensory defects caused by Pseudomonas (Bassette et al., 1986). Future experiments are needed to confirm the specific compounds and pathways that are responsible for the development of this color defect.

Our data clearly show that the $P$. azotoformans strain isolated here is responsible for gray color development when present in refrigerated, pasteurized milk in partially filled containers. This finding is important because the defect would likely remain undetected in unopened containers (representing the samples typically used for shelf-life testing by the dairy industry) but may be detected by consumers after partial use of the milk. The fact that the gray color-generating ability of $P$. azotoformans can go undetected in routine fluid milk shelf-life testing is consistent with the fact that the case investigated here was initiated from a consumer complaint. Our report expands the range of defects and spoilage issues that are linked to the presence of Pseudomonas species in fluid milk and other dairy products. We show that color defects, in addition to the well-described blue color that can be produced

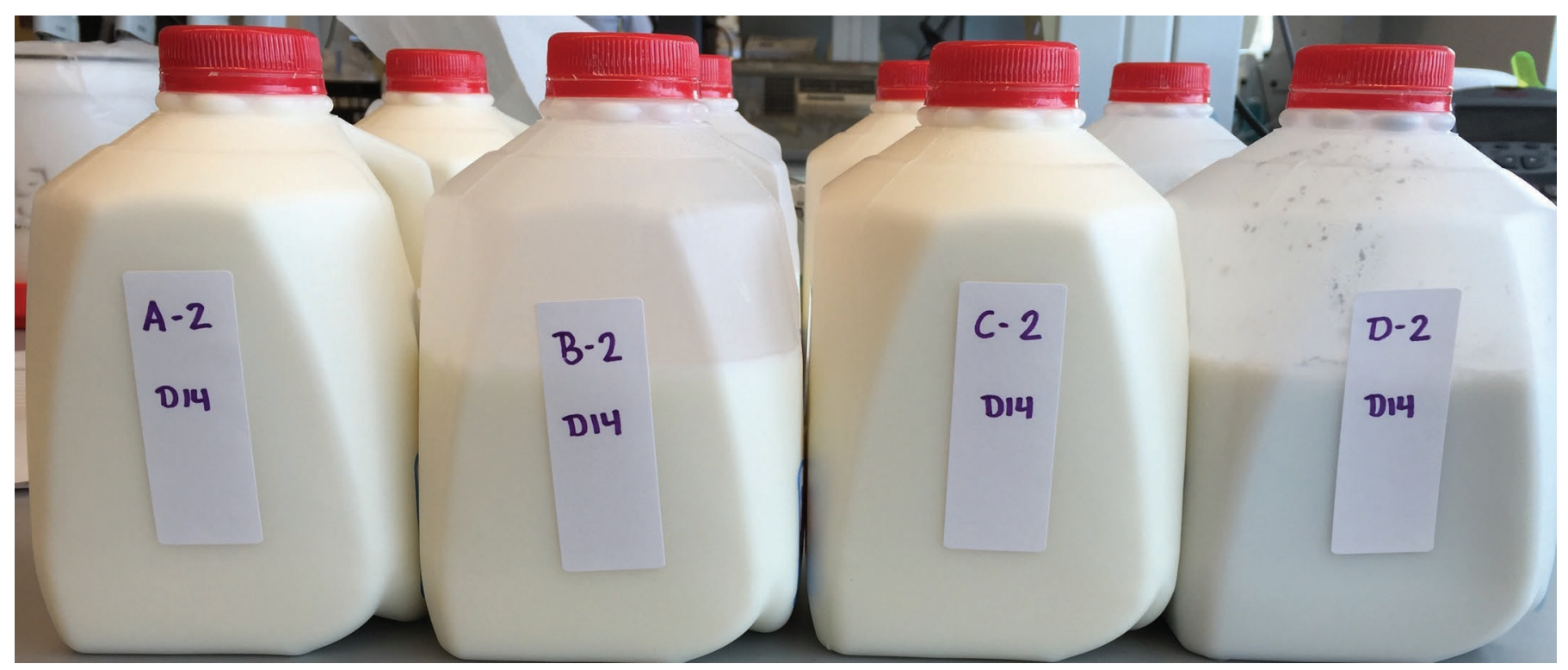

Figure 1. Color development in milk samples inoculated with Pseudomonas azotoformans (FSL E2-0548) and corresponding uninoculated samples, all incubated for $14 \mathrm{~d}$ at $6^{\circ} \mathrm{C}$. From left to right: (A) uninoculated "full" quart-size container with original milk volume; (B) uninoculated "half-full" quart-size container; (C) inoculated "full" quart-size container; and (D) inoculated "half-full" quart-size container. 
Sample Type

- Control

.... Inoculated

A
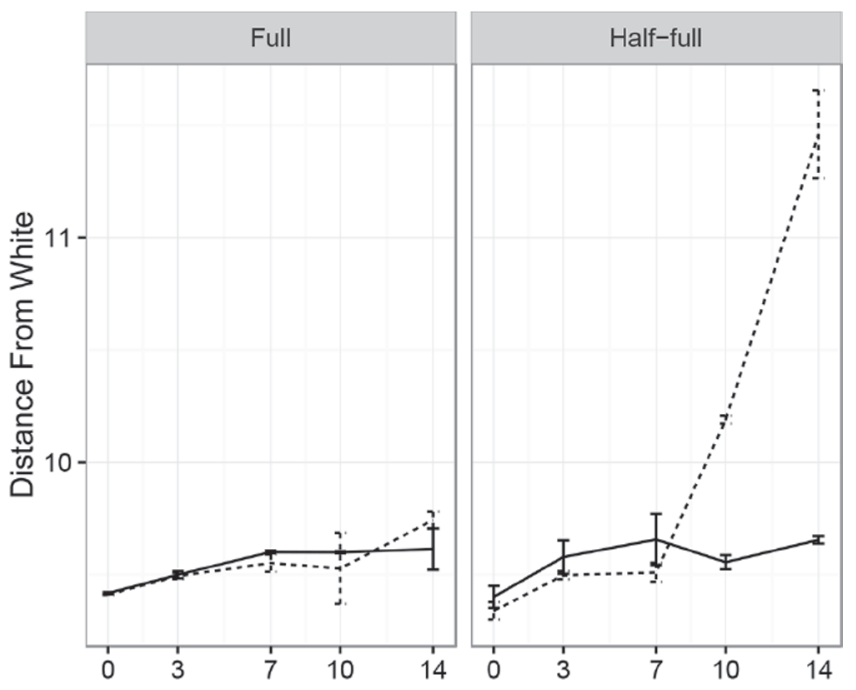

B

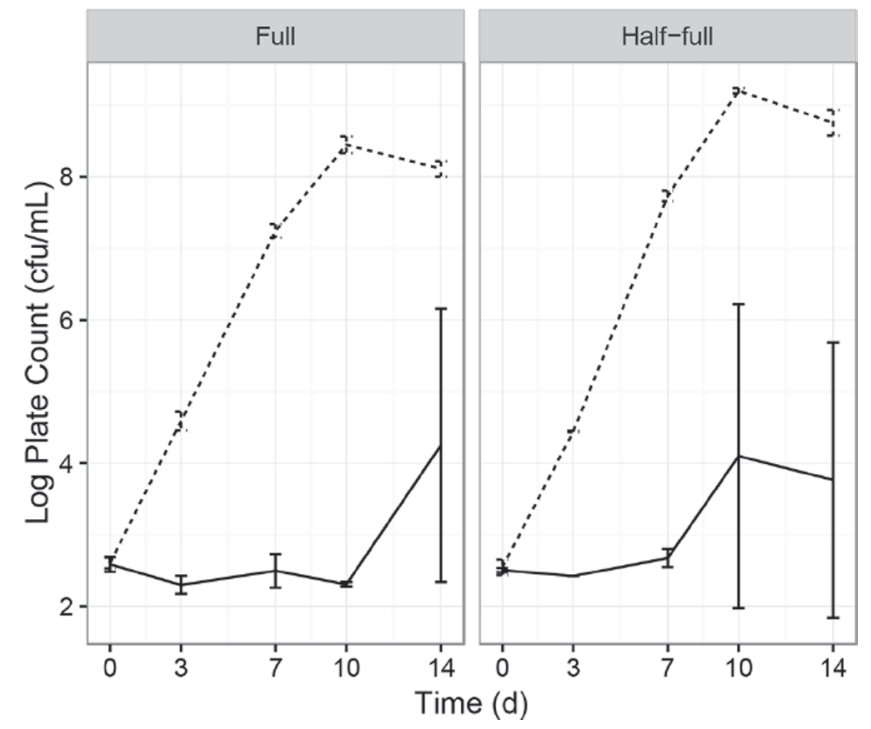

Figure 2. (A) Color (expressed as distance from white), and (B) bacterial numbers $(\log \mathrm{cfu} / \mathrm{mL})$ in fluid milk samples over $14 \mathrm{~d}$ of incubation at $6^{\circ} \mathrm{C}$ with measurements taken on $\mathrm{d} 0,3,7,10$, and 14. Values represent the averages of 2 independent biological replicates; error bars represent standard deviations. At d 10, 1 uninoculated sample had bacterial numbers of $>400,000 \mathrm{cfu} / \mathrm{mL}$ and at $\mathrm{d} 14$, 2 uninoculated samples each showed bacterial numbers of $>400,000$ $\mathrm{cfu} / \mathrm{mL}$, leading to large standard deviations on these days. These findings suggest inherent sporadic post-pasteurization contamination with psychrotolerant organisms in the milk used for our experiments, which is not unusual in HTST-pasteurized milk. Although this could also be caused by psychrotolerant spore-forming bacteria that survived pasteurization, all samples were from the same batch of raw milk and only 1 of the 4 control bottles tested on d 10, and 2 of the 4 control bottles tested on d 14 showed high-level growth, which is consistent with sporadic post-pasteurization contamination. by certain Pseudomonas strains, occur (Price-Whelan et al., 2007; Martin et al., 2011). This information is important to dairy processors because they might not associate unexpected black or gray colors in dairy products with the presence of microbial contaminants, but rather, with tampering (e.g., addition of ink) or other inadvertent chemical contamination.

\section{ACKNOWLEDGMENTS}

This project was supported by the New York State Dairy Promotion Advisory Board, which embodies New York dairy farmers' commitment to production of highquality dairy products, through the New York State Department of Agriculture and Markets (Albany, NY). The authors thank the staff and students of the Milk Quality Improvement Program (Cornell University, Ithaca, NY) and the Food Safety Laboratory (Cornell University, Ithaca, NY) for assistance with this study.

\section{REFERENCES}

Bassette, R., D. Y. C. Fung, V. R. Mantha, and E. H. Marth. 1986. Off-flavors in milk. Crit. Rev. Food Sci. Nutr. 24:1-52.

Cantoni, C., S. Stella, M. Cozzi, L. Iacumin, and G. Comi. 2003. Blue colouring in mozzarella cheese. Indust. Aliment. 42:840-843.

Dogan, B., and K. J. Boor. 2003. Genetic diversity and spoilage potentials among Pseudomonas spp. isolated from fluid milk products and dairy processing plants. Appl. Environ. Microbiol. 69:130-138. https://doi.org/10.1128/AEM.69.1.130-138.2003.

Huck, J. R., N. H. Woodcock, R. D. Ralyea, and K. J. Boor. 2007. Molecular subtyping and characterization of psychrotolerant endospore-forming bacteria in two New York State fluid milk processing systems. J. Food Prot. 70:2354-2364.

Laird, D. T., F. M. Gambrel-Lenarz, T. E. Graham, and R. Reddy. 2004. Standard Methods for the Examination of Dairy Products. 17th ed. H. Wehr and J. F. Frank, ed. American Public Health Association, Washington, DC.

Lenth, R. V. 2016. Least-squares means: The R Package lsmeans. J. Stat. Softw. 69:1-33. https://doi.org/10.18637/jss.v069.i01.

Martin, N. H., S. C. Murphy, R. D. Ralyea, M. Wiedmann, and K. J. Boor. 2011. When cheese gets the blues: Pseudomonas fluorescens as the causative agent of cheese spoilage. J. Dairy Sci. 94:31763183. https://doi.org/10.3168/jds.2011-4312.

Palleroni, N. J. 1984. Pseudomonas. Vol. 1. Williams \& Wilkins, Baltimore, MD.

Price-Whelan, A., L. E. P. Dietrich, and D. K. Newman. 2007. Pyocyanin alters redox homeostasis and carbon flux through central metabolic pathways in Pseudomonas aeruginosa PA14. J. Bacteriol. 189:6372-6381. https://doi.org/10.1128/JB.00505-07.

$\mathrm{R}$ Core Team. 2016. R: A language and environment for statistical computing. R Core Team, Vienna, Austria.

Stellato, G., D. R. Utter, A. Voorhis, M. De Angelis, A. M. Eren, and D. Ercolini. 2017. A Few Pseudomonas oligotypes dominate in the meat and dairy processing environment. Front. Microbiol. 8:264 https://doi.org/10.3389/fmicb.2017.00264.

Wickham, H. 2009. ggplot2: Elegant Graphics for Data Analysis. Springer-Verlag, New York, NY.

Yam, K. L., and S. E. Papadakis. 2004. A simple digital imaging method for measuring and analyzing color of food surfaces. J. Food Eng. 61:137-142. https://doi.org/10.1016/S0260-8774(03)00195-X. 